\title{
A NOTE ON THE BRAUER GROUP
}

\section{ALAN McCONNELL}

1. Introduction. Let $F$ be a field, $K$ an extension field; let $B(K / F)$ denote the Brauer group of classes of simple algebras with center $F$ split by $K . B(K / F)$ is a functor in both $K$ and $F$ which is "left exact" in $K$ : if $L$ is an extension of $K$, the injection $K \rightarrow L$ induces an injection $B(K / F) \rightarrow B(L / F)$.

Now assume that $F$ is a field of characteristic $p>0$, and let $C$ be a purely inseparable, $K_{s}$ a separable extension of $F$, both finite. It is well known that $C \otimes_{F} K_{s}$ is a field, which we shall denote by $K ; K$ is the direct sum ("inverse product") of $C$ and $K_{s}$ in the category of finite extension fields of $F$. One might expect that since $B(/ F)$ is left exact, this property of $K$ is reflected in $B(K / F)$. Indeed, if $\left[K_{s}: F\right]$ and $[C: F]$ are relatively prime, one sees easily that $B(K / F)$ is the direct sum of $B(C / F)$ and $B\left(K_{s} / F\right)$. However, if $\left[K_{s}: F\right]$ is also a $p$ th power, $B(C / F)$ and $B\left(K_{s} / F\right)$ will in general have a nontrivial intersection; an algebra class over $F$ can be split by both $C$ and $K_{s}$. The question arises: is $B(K / F)$ generated by its subgroups $B(C / F)$ and $B\left(K_{8} / F\right)$ ? The purpose of this note is to give an example which answers this question in the negative.

2. The example. As in the introduction, let $F$ be a field of characteristic $p>0$, and let $C=F(\eta)$ where $\eta^{p} \in F, \eta \notin F$; assume further that $C^{p}=F$. Let $K_{s}$ be a cyclic extension of $F,\left[K_{s}: F\right]=p$. Let $K=K_{s} \otimes C$ be the composite extension field. Finally, we assume that there exists a division algebra $D$ with center $F$ and maximal commutative subfield $K$. The construction of a specific $F, C, K_{s}$ and $D$ will be done in the 3rd section. The Brauer class of $D,[D]$, is thus an element of $B(K / F)$.

Theorem. $[D] \in B(K / F)$ is not the product of $\alpha \in B\left(K_{s} / F\right)$ and $\beta \in B(C / F)$.

Proof. Suppose $[D]=\alpha \cdot \beta$; we derive a contradiction. Let $A \in \alpha$ be central simple/ $F$ with maximal commutative subfield $K_{s}, B \in \beta$ have $C$ as maximal commutative subfield [1, Theorem 4.27, p. 61]. We note that neither $\alpha$ nor $\beta$ is the identity element of $B(K / F)$, since $D$ is not split by $C$ or $K_{8}$. We have $[A: F]=p^{2}=[B: F]$, and since neither $A$ nor $B$ are matrices over $F$, they must both be division alge-

Received by the editors February 24, 1966.

1 This work was supported by NSF Grant GP-5276. 
bras [1, Theorem 3.18 , p. 43 ]. By hypothesis $A \otimes B \cong D \otimes \mathbb{M}$ where $\mathfrak{T C}$ is a full matrix ring over $F$; however, a dimension count shows $\Re=F$, and $A \otimes B \cong D$. Thus we can consider $A$ and $B$ as division subalgebras of $D$, both with center $F$ and each the centralizer of the other [1, Theorem 4.13, p. 53].

Now $A$ is a cross-product, indeed a cyclic algebra: $A=K_{s} \oplus y K_{s} \oplus$ $\cdots \oplus y^{p-1} K_{s}$, where $y$ satisfies the following properties: $y^{-1} k y$ $=k^{\sigma}\left(\sigma \in \mathcal{G}\left(K_{s} / F\right), \sigma \neq 1\right)$ and $y^{p} \in F[1$, p. 74]. Considered as an element of $D, y$ must commute with $B$ and hence with $C \subset B$. Thus $C(y)$ is a field contained in $D$. On the one hand, $C(y)$ must be a bigger field than $C$, since if $y$ were in $C$ it would commute with $K_{s}$; on the other hand, since $y^{p} \in F$ and $C^{p}=F, y \in C(y)$ must be in $C$. This contradiction proves the theorem.

3. Construction of $D$. We must exhibit: a field $F$, a division algebra $D$ with center $F$ and degree $p^{2}$ over $F$ (i.e. $[D: F]=p^{4}$ ); a subfield of $D, K_{s}$, containing $F$ and cyclic over $F$; a field $C$ such that $F \subset C \subset D$ and $C^{p}=F$, such that $C$ and $K_{s}$ commute elementwise.

Let $F=G F(p)(\pi)$ where $\pi$ is an indeterminate over $G F(p)$. Let $R$ be the (unique) cyclic extension of $G F(p)$ of degree $p^{2}$. Set $L=R(\pi)$; $L$ is a cyclic extension of $F$ of degree $p^{2}$. Let $\sigma$ be the generating automorphism of $\mathfrak{g}(L / F) ; \sigma^{p^{2}}=1$. Finally, form $D=(L, \sigma, \pi)[1$, p. 74].

One sees immediately via polynomial degree considerations that the indeterminate $\pi$ is not a norm from $K_{s}=\left\{k \in L: k^{\sigma^{p}}=k\right\}$. This suffices to show that $D$ is a division algebra $[1$, Theorem 7.19, p. 98]. Note that the field $K_{s}$ is cyclic of degree $p$ over $F$.

We construct $C$ as follows: by definition of $D$ there exists an element $y \in D$ such that $y^{p^{2}}=\pi$ ( $y$ induces the automorphism $\sigma$ in $L \subset D=(L, \sigma, \pi))$. Let $C=F\left(y^{p}\right)$; then, since $\left(y^{p}\right)^{p}=\pi, C^{p}=F$. Note that $C$ and $K_{s}$ commute, since $y^{p}$ and $K$, do; thus $K=C \otimes K_{s}$ is a subfield of $D$.

\section{BIBLIOGRAPHY}

1. A. A. Albert, Structure of algebras, rev. ed., Amer. Math. Soc. Colloq. Publ. Vol. 24, Amer. Math. Soc., Providence, R.I., 1961.

University of Illinois, Chicago 